\title{
Lack of Critical Phase Points and Exponentially Faint Illumination
}

Franco Cardin (cardin@math.unipd.it) and Alberto Lovison

(lovison@math.unipd.it)

Dipartimento di Matematica Pura ed Applicata

Via Belzoni, 7 - 35131 Padova (Italy)

\begin{abstract}
The Stationary Phase Principle (S.P.P.) states that in the computation of oscillatory integrals, the contributions of non stationary points of the phase are smaller than any power $n$ of $1 / k$, for $k \rightarrow \infty$. Unfortunately, S.P.P. says nothing about the possible growth in the constants in the estimates with respect to the powers $n$. A quantitative estimate of oscillatory integrals with amplitude and phase in the Gevrey classes of functions shows that these contributions are asymptotically negligible, like $\exp \left(-a k^{b}\right), a, b>0$. An example in Optics is given.
\end{abstract}

Keywords: Stationary Phase, Oscillatory Integrals, Wave Optics, Symplectic Geometry, Lagrangian Submanifolds

\section{Introduction}

An oscillatory integral is an integral of the form

$$
I(k):=\int_{u \in \Omega} a(u) e^{-i k \varphi(u)} d u, \quad \Omega \subseteq \mathbb{R}^{d},
$$

where $a$ and $\varphi$ are $C^{\infty}$ real functions, called respectively amplitude and phase, and $k$ is a (large) parameter. They are typically employed to represent solutions for linear PDE's depending on a real parameter, e.g. the Schrödinger equation or the Helmholtz equation.

A well known feature is the tight dependence on the values of $a$ near the critical points of $\varphi$. More precisely, if in the domain of integration $\Omega$ there are no degenerate critical points for $\varphi$, the Stationary Phase Principle $^{1}$ holds, i.e.

$$
I(k) \simeq\left(\frac{2 \pi}{k}\right)^{\frac{d}{2}} \sum_{u_{0}: \nabla \varphi\left(u_{0}\right)=0} a\left(u_{0}\right) \exp \left\{i k \varphi\left(u_{0}\right)\right\} \frac{e^{i \frac{\pi}{4} \operatorname{sgn}\left(\nabla^{2} \varphi\left(u_{0}\right)\right)}}{\sqrt{\operatorname{det} \nabla^{2} \varphi\left(u_{0}\right)}} .
$$

In particular, a very standard argument, states that the contributions to $I$ coming from a compact subset $K \subset \Omega$ where there are no

1 This short-wave approximation is usually referred in physics as the WKB method, and it seems that it was first worked out by F. Carlini [4] -we learned it in [1]-, and later used by Kelvin, Stokes and many others in the 19th century.

(C) 2004 Kluwer Academic Publishers. Printed in the Netherlands. 
stationary points, tends to zero faster than every positive power of $\frac{1}{k}$, as $k \rightarrow \infty$.

This "superpolynomial estimate" can be written as

$$
|I(k)| \leq A_{n} k^{-n}, \quad \forall n \in \mathbb{N},
$$

(and will be revisited in Theorem 1). The coefficients $A_{n}$ are comparable to the size of the $n$-th order derivatives of the amplitude function $a$. Unfortunately, for $C^{\infty}$ compactly supported functions there is no $a$ priori upper bound to the growth of these derivatives .

If the amplitude were analytic, it would be possible to estimate $A_{n}$ by $n$ !. In that case, choosing an optimal value of $n$ depending on $k$ and applying Stirling's formula, as shown in detail in the next section, one would find the expected exponential estimate for $I$ :

$$
|I(k)| \leq n ! k^{-n}, \forall n \in \mathbb{N}, \quad \Rightarrow \quad|I(k)| \leq A e^{-k} .
$$

Unfortunately, analyticity is a requirement which cannot be satisfied by a non trivial compactly supported function. On the other hand, in order to have simpler integrals to manage, we choose to consider compactly supported amplitudes, as done, e.g., in [2], although it would be possible to discuss the Stationary Phase Principle for non compactly supported amplitudes.

A reasonable way out can be found by turning to an intermediate class of functions placed between the spaces of the $C^{\infty}$ functions and the analytic functions: the Gevrey spaces.

Functions in the Gevrey space $G^{s}(\Omega), s \geq 1$ satisfy the inequality:

$$
\left|\partial^{\alpha} f(u)\right| \leq C^{|\alpha|+1}(\alpha !)^{s}
$$

for every $\alpha=\left(\alpha_{1}, \ldots, \alpha_{d}\right) \in \mathbb{N}^{d}$, for every $u$ in a compact subset $K \Subset \Omega$, and a suitable constant $C$, depending only on $K$.

Obviously, $G^{1}(\Omega)$ is the class of the analytic functions $\mathcal{A}(\Omega)$. It is also clear that there exist $C^{\infty}$ functions which are not Gevrey for any $s$. To summarize, one has

$$
\mathcal{A}(\Omega) \varsubsetneqq G^{s}(\Omega) \varsubsetneqq C^{\infty}(\Omega), \quad s \in \mathbb{R}, s>1 .
$$

Let $G_{0}^{s}(\Omega)$ denote the space of complactly supported " $s$-Gevrey" functions in $\Omega$. Gevrey spaces $G_{0}^{s}(\Omega)$, with $s>1$, are dense in $C_{0}^{\infty}(\Omega)$, in $L_{0}^{p}(\Omega)$, etc., essentially because partitions of unity can be constructed. See [8] for an exhaustive treatise.

When $a$ belongs to $G_{0}^{s}(\Omega)$, and $\varphi$ to $G^{s}(\Omega)$, we obtain the exponential estimate:

$$
|I(k)| \leq A \sqrt{k} e^{-\sigma k^{\frac{1}{s}}}, \quad k \geq 1 .
$$


This result, allowing $\varphi$ to belong to a Gevrey class of functions, improves a previous one by Todor Gramchev for analytical phases $\varphi$, see $[6]$.

\section{A Gevrey exponentially decreasing estimate}

The first theorem is standard; the second is the core of the main result, which we state as the third theorem. From now on we will assume $\Omega$ bounded.

THEOREM 1. Let $a \in C_{0}^{\infty}(\Omega), \varphi \in C^{\infty}(\Omega), \frac{\partial \varphi}{\partial u} \neq 0, \forall u \in \operatorname{supp} a$. Then

$$
|I(k)| \leq A_{n} k^{-n}, \quad \forall n \in \mathbb{N}, \quad k \geq 1 .
$$

THEOREM 2. Let $a \in G_{0}^{s}(\Omega), \varphi=u_{1}$, then, for suitable constants $A$ and $\sigma$,

$$
|I(k)| \leq A \sqrt{k} e^{-\sigma k^{\frac{1}{s}}}, \quad k \geq 1 .
$$

This assertion is also true when $\varphi$ is an arbitrary Gevrey function, in particular analytic, i.e. when $\varphi \in G^{s}(\Omega), s \geq 1$.

THEOREM 3. Let $a \in G_{0}^{s}(\Omega)$ and $\varphi$ be analytic or belonging to $G^{s}(\Omega)$, with $\frac{\partial \varphi}{\partial u} \neq 0, \forall u \in \operatorname{supp} a$. Then, for suitable constants $A$ and $\sigma$,

$$
|I(k)| \leq A \sqrt{k} e^{-\sigma k^{\frac{1}{s}}}, \quad k \geq 1 .
$$

REMARK 4. In theorems (2) and (3) occurs a $\sqrt{k}$, coming from the Stirling formula, which could be absorbed by the exponential decay, by little increment of the constant $\sigma$. We keep on writing it since $A$ and $\sigma$ depend explicitly, as shown below, on the Gevrey constants $C$ and $s$ in (5), for the amplitude $a$ and the phase $\varphi$.

Proof of Theorem 1. Following [2], we claim that there is no loss of generality if we assume $\varphi\left(u_{1}, \ldots, u_{d}\right)=u_{1}$.

First consider an open covering $\left\{U_{l}\right\}$ of suppa, such that in each $U_{l}$ there is at least one non vanishing partial derivative of $\varphi$. Then, by means of a subordinate partition of unity $\left(\sum_{\iota \in \Upsilon} \theta_{\iota}=1, \operatorname{supp} \theta_{\iota} \subseteq U_{l}\right.$, for some $l$ ), we can decompose the original integral as follows:

$$
\int_{\operatorname{supp} a} a e^{i k \varphi}=\sum_{\iota \in \Upsilon} \int a \theta_{\iota} e^{i k \varphi}=\sum_{\iota \in \Upsilon} \int_{\operatorname{supp} a_{\iota}} a_{\iota} e^{i k \varphi} .
$$

Since supp $a$ is compact by hypothesis, $\Upsilon$ can be chosen finite. We now show that each of these integrals can be transformed into an integral of the desired form by means of a suitable change of variables. 
By reordering variables and changing the orientation if needed, one can obtain $\frac{\partial \varphi}{\partial u_{1}}>0$. The map

$$
u=\left(u_{1}, \ldots, u_{d}\right) \stackrel{\eta}{\longmapsto} v=\left(\varphi(u), u_{2}, \ldots, u_{d}\right),
$$

is clearly a globally invertible diffeomorphism in every open convex subset of $U$. Then the inverse map $\xi:=\eta^{-1}$ fits our purpose. Indeed:

$$
\begin{aligned}
I(k) & =\int_{\Omega} a(u) e^{i k \varphi(u)} d u=\int_{\widetilde{\Omega}} a(\xi(v)) e^{i k \varphi(\xi(v))}\left|J_{\xi}(v)\right| d v= \\
& =\int_{\widetilde{\Omega}} a(\xi(v))\left|\frac{\partial \varphi}{\partial u_{1}}(\xi(v))\right|^{-1} e^{i k v_{1}} d v=\int_{\widetilde{\Omega}} \tilde{a}(v) e^{i k v_{1}} d v .
\end{aligned}
$$

Now we prove the main fact. For every fixed $(d-1)$-tuple $\left(u_{2}, \ldots, u_{d}\right)$, let

$$
u_{1 m}\left(\text { resp. } u_{1 M}\right):=\inf (\text { resp. } \sup )\left\{u_{1} \mid\left(u_{1}, u_{2}, \ldots, u_{d}\right) \in \Omega\right\} .
$$

Hence,

$$
\begin{array}{r}
I(k)=\int a(u) e^{i k u_{1}} d u=\iint_{u_{1 m}}^{u_{1 M}} a(u) \frac{1}{i k} \frac{\partial}{\partial u_{1}}\left(e^{i k u_{1}}\right) d u_{1} d u_{2} \ldots d u_{d}= \\
=\int\left\{\left[a(u) \frac{1}{i k} e^{i k u_{1}}\right]_{u_{1 m}}^{u_{1 M}}-\frac{1}{i k} \int_{u_{1 m}}^{u_{1 M}} \frac{\partial a(u)}{\partial u_{1}} e^{i k u_{1}} d u_{1}\right\} d u_{2} \ldots d u_{d}= \\
=-\frac{1}{i k} \int_{\Omega} \frac{\partial a(u)}{\partial u_{1}} e^{i k u_{1}} d u .
\end{array}
$$

Performing $n$ times this procedure and taking absolute values gives:

$$
\begin{array}{r}
|I(k)|=\left(\frac{1}{k}\right)^{n}\left|\int_{\Omega}\left(\frac{\partial^{n} a}{\partial u_{1}^{n}}\right) e^{i k u_{1}} d u\right| \leq \\
\leq\left(\frac{1}{k}\right)^{n} \int_{\Omega}\left|\left(\frac{\partial^{n} a}{\partial u_{1}^{n}}\right)\right| d u=: A_{n} k^{-n},
\end{array}
$$

as desired.

Proof of Theorem 2. The hypotheses of this statement are exactly the hypotheses of the reduced case of the preceding theorem, apart from the stronger hypotheses on the amplitude. A straightforward substitution of (5) in the inequality of (15) gives

$$
A_{n} \leq \operatorname{meas}(\Omega) c^{n+1}(n !)^{s},
$$

with $c=C(\bar{\Omega})$, so for the integral we can write, setting $B=$ meas $(\Omega) c$,

$$
|I(k)| \leq B k^{-n} c^{n}(n !)^{s}=B\left(\frac{c}{k}^{\frac{n}{s}} n !\right)^{s}, \quad \forall n \in \mathbb{N}
$$


which, by Stirling's formula:

$$
n !=n^{n} e^{-n} \sqrt{2 \pi n} e^{\frac{\theta(n)}{12 n}}, \quad \text { where } 0<\theta(n)<1,
$$

becomes

$$
\begin{aligned}
& =B\left(\left(\frac{c}{k}\right)^{\frac{n}{s}} n^{n} e^{-n} \sqrt{2 \pi n} e^{\frac{\theta(n)}{12 n}}\right)^{s}, \\
& =B\left(\left(\left(\frac{c}{k}\right)^{\frac{1}{s}} n\right)^{n} e^{-n} \sqrt{2 \pi n} e^{\frac{\theta(n)}{12 n}}\right)^{s} .
\end{aligned}
$$

Note that $e^{\frac{\theta(n)}{12 n}}$ is surely smaller than 2 for all $n \geq 1$, and choose $n$ in order to bound the quantity in the inner parentheses by 1 , i.e. set $n^{\star}:=n^{\star}(k)=$ (integer part of $)\left[\left(\frac{k}{c}\right)^{\frac{1}{s}}\right]$. Hence

$$
|I| \leq 2 B e^{-s\left(\frac{k}{c}\right)^{\frac{1}{s}}} \sqrt{(2 \pi)^{s} \frac{k}{c}}=A \sqrt{k} e^{-\sigma k^{\frac{1}{s}}}
$$

where

$$
\begin{gathered}
A=2 B \sqrt{\frac{(2 \pi)^{s}}{c}} \\
\sigma=s c^{-\frac{1}{s}} .
\end{gathered}
$$

Proof of Theorem 3. In order to reduce this statement to Theorem 2 , we need to prove that the amplitude obtained performing a change of variables as in (13):

$$
\tilde{a}(v)=a(\xi(v))\left|\frac{\partial \varphi}{\partial u_{1}}(\xi(v))\right|^{-1},
$$

is a Gevrey function as long as $a(u)$ and $\varphi(u)$ are. Hence we need to recall the following facts: if $\varphi \in G^{s}(\Omega)$,

(i) $\xi:=\eta^{-1}$, where $\eta(u)=\left(\varphi(u), u_{2}, \ldots, u_{d}\right)$ as in (12), is a $G^{s}$ diffeomorphism.

(ii) $1 /\left(\frac{\partial \varphi}{\partial u_{1}}(u)\right) \in G^{s}(\Omega)$.

(iii) The composition of a Gevrey function with a Gevrey diffeomorphism is still a Gevrey function of the same order $s$.

These are all known facts, a proof of which can be found in [7], where these results are proved in more general spaces of functions. Alternatively, one can find a proof which is specifically suitable for our purpose in [5]. 


\section{An application to Optics}

Consider the propagation of the light in the plane $\mathbb{R}^{2}$ due to a compact and regular emitting surface $\Sigma$ (i.e. a closed simple curve), or, equivalently, consider a light emission for which such a $\Sigma$ is a surface of constant phase. For monochromatic propagation of wave number $k$, the evolution is governed by the 'Helmholtz equation', which entails for the phase function to satisfy an Hamilton-Jacobi type equation: the eikonal equation. For isotropic homogeneous media (constant refractive index), the eikonal equation takes the form:

$$
|\nabla \varphi(x)|^{2}=1 .
$$

A complete integral for this equation is given by

$$
\varphi(x, \theta):=x \cdot n(\theta),
$$

where $n: \mathbb{S}^{1} \rightarrow \mathbb{R}^{2}, \theta \mapsto(\cos (\theta), \sin (\theta))$. Suppose the initial datum be $\sigma$ given on $\Sigma$, which we think as parametrized by $\mathbb{S}^{1} \ni \chi \mapsto x(\chi) \in \mathbb{R}^{2}$. It is well known that in the general case no global classical solution exists. However, the modern geometric Hamilton-Jacobi theory always admits globally defined solutions. ${ }^{2}$ The solution of the Geometrical Cauchy Problem is a Lagrangian submanifold $\Lambda \subset T^{\star} \mathbb{R}^{2}$, globally generated by the Morse Family, (i.e. generating function) $\tilde{\varphi}$ :

$$
\begin{gathered}
\tilde{\varphi}(x ; \theta, \chi):=(x-x(\chi)) \cdot n(\theta)+\sigma(\chi), \\
\Lambda=\left\{(x, p): p=\frac{\partial \tilde{\varphi}}{\partial x}, d_{u} \tilde{\varphi}=0, i . e .\left\{\begin{array}{c}
\frac{\partial \tilde{\varphi}}{\partial \theta}=0 . \\
\frac{\partial \tilde{\varphi}}{\partial \chi}=0 .
\end{array}\right\}\right.
\end{gathered}
$$

In such a case, the auxiliary variables $u=(\theta, \chi)$ belong to $\mathbb{S}^{1} \times \mathbb{S}^{1}=\mathbb{T}^{2}$. The amplitude of light observed in a point $x$ of the plane is then given by an oscillatory integral, extended over the parameter space $\mathbb{T}^{2}$ :

$$
I(x ; k)=\left(\frac{2 \pi}{k}\right)^{2} \int_{\mathbb{T}^{2}} a(x ; \chi, \theta) e^{i k \tilde{\varphi}(x ; \theta, \chi)} d \theta d \chi .
$$

Here the amplitude function $a(x ; \theta, \chi)$ satisfies the 'transport equations' because $I(x ; k)$ is a solution of the Helmholtz equation.

Stationary Phase states that the only relevant contributions in the asymptotic expression of $I(x ; k)$ come from arbitrarily small neighbourhoods of the $u$-critical points of $\tilde{\varphi}$. They are given by

$$
d_{u} \tilde{\varphi}=0:\left\{\begin{array} { l } 
{ \frac { \partial \tilde { \varphi } } { \partial \theta } = 0 , } \\
{ \frac { \partial \tilde { \varphi } } { \partial \chi } = 0 . }
\end{array} \Leftrightarrow \left\{\begin{array}{l}
-n(\theta) \cdot \frac{\partial x}{\partial \chi}(\chi)=0, \\
\frac{\partial n}{\partial \theta}(\theta) \cdot(x-x(\chi))=0 .
\end{array}\right.\right.
$$

\footnotetext{
${ }^{2}$ See e.g. $[1,3]$ and the bibliography quoted therein.
} 

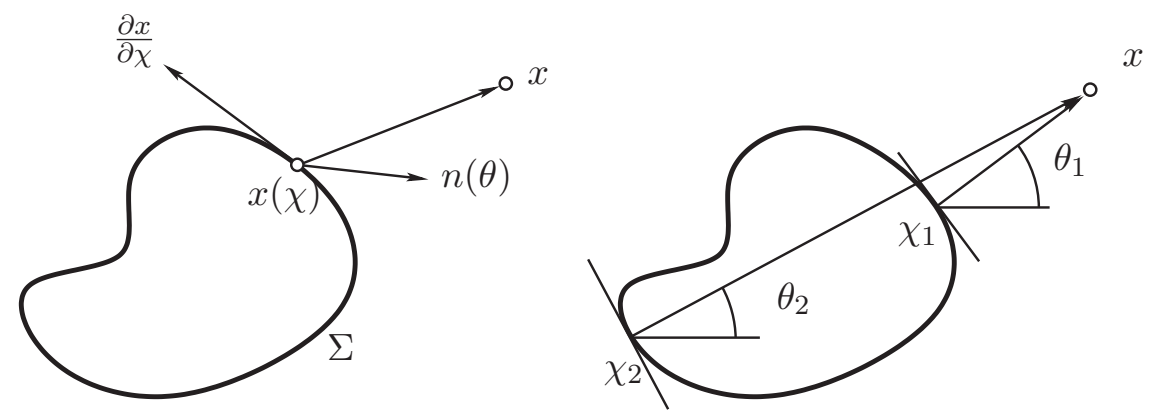

Figure 1. Light emission from a surface $\Sigma$

From (a) one obtains that $n$ must be $\perp$ to the emitting surface $\Sigma$, from (b) one obtains that $x-x(\chi)$ must be parallel to $n$. In other words, $u_{0}=\left(\theta_{0}, \chi_{0}\right)$ is a critical point for $\tilde{\varphi}$ if and only if $\chi_{0}$ is a critical point for the distance function between $x$ and $\Sigma$ and $n(\theta)$ is parallel to the connecting vector (see Figure 1).

It is self-explanatory that this is a wave translation of the Fermat Principle of geometrical optics.

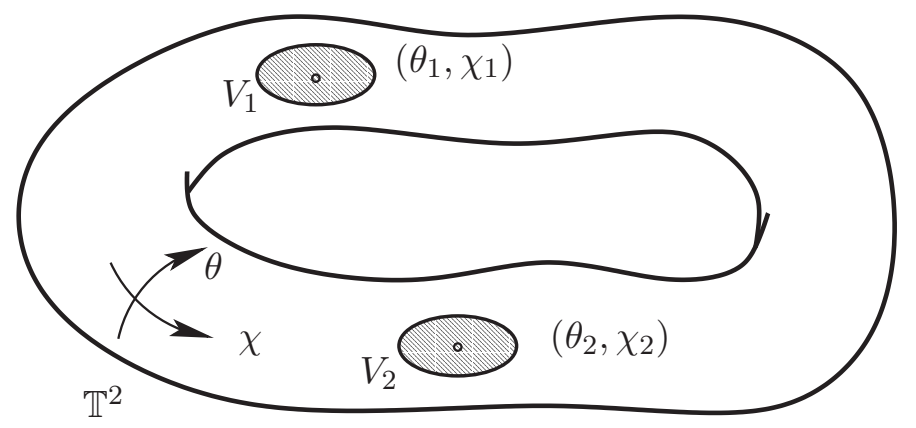

Figure 2. The domain of integration of the "reduced integral" of the example.

In order to exhibit an application of our result, fix an observation point $x$ in the plane, and consider a Gevrey amplitude function $a(x ; \theta, \chi)$, possibly obtained by means of a Gevrey partition of unity, which vanishes in a neighbourhood $V$ of the critical points $u_{\iota}=\left(\theta_{\iota}, \chi_{\iota}\right)$ of the phase. (See $V=V_{1} \cup V_{2}$ in Figure 2.)

Thus, our result asserts that the oscillatory integral so obtained, is exponentially vanishing as $k$ tends to infinity, as is the illumination at $x$, which is represented by this reduced integral. 


\section{Acknowledgements}

The authors gratefully thank Todor Gramchev, Luigi Rodino and Giuseppe Zampieri for fruitful suggestions and exchanges of ideas.

\section{References}

1. V.I. Arnol'd, Symplectic geometry and topology, Journal of Math. Physics 41, n.6 (2000), 3307-3343.

2. V.I. Arnol'd, A.N. Varchenko, S.M. Gusein-Zade, Singularities of Differentiable Maps, Birkhäuser, 1985.

3. F. Cardin, The global finite structure of generic envelope loci for HamiltonJacobi equations, Journal of Math. Physics 43, n.1 (2002), 417-430.

4. F. Carlini, Ricerche sulla convergenza della serie che serve alla soluzione del Problema di Keplero, Schumacher Astronomische Nachrichten 28, 257-270; 30, 197-254; (1817).

5. T. Gramchev, Implicit function theorem in the Gevrey classes, preprint, 2002.

6. T. Gramchev, The stationary phase method in Gevrey classes and Fourier Integral Operators on Ultradistributions, in: Partial Differential Equations, Banach Cent. Publ. 19, Warsaw (1987), 101-112.

7. J.L. Lions and E. Magenes, Problèmes aux limites non homogènes et applications, Vol. 3, Dunod, Paris, 1970.

8. L. Rodino, Linear Partial Differential Operators in Gevrey Spaces, World Scientific, Singapore, 1993. 